\title{
ON THE HEIGHTS OF GROUP CHARACTERS
}

\author{
R. J. HAGGARTY
}

\begin{abstract}
ABSTRACr. For a finite $p$-soluble group $G$ we derive a bound on the heights of the irreducible complex characters of $G$ lying in a $p$-block $B$. This bound depends on the prime $p$ and the exponent $d$ of a defect group of $B$. We show by examples that this bound is of the right order of magnitude.
\end{abstract}

Let $G$ be a finite group of order $p^{e} g_{0}$, where $p$ is a fixed prime, $e$ is an integer $\geqslant 0$, and $\left(g_{0}, p\right)=1$. In the theory of modular representations, the characters of the irreducible complex representations of $G$ may be partitioned into disjoint sets, the so-called blocks of $G$ for the prime $p$. Associated with each block $B$ is a $p$-subgroup $D$ of $G$ of order $p^{d}$, unique up to conjugacy in $G$. $d$ is called the defect of the block $B$. If $\chi$ is an irreducible complex character of $G$, or as we shall say, an ordinary character of $G$, and $\chi$ lies in a block $B$, written $\chi \in B$, then $\chi$ has degree divisible by $p$ to the exponent $(e-d+h(\chi))$. The nonnegative integer $h(\chi)$ is called the height of $\chi$.

In [4] Fong proves the following: Let $G$ be a finite $p$-soluble group and $B$ be a block of $G$ for the prime $p$. Suppose that $B$ has defect group $D$ and let $Z(D)$ denote the centre of $D$. Then for each ordinary character $\chi \in B$ we have $h(\chi) \leqslant \nu_{p}(|D: Z(D)|)$ where $\nu_{p}(t)$ denotes the exponent of $p$ dividing $t$.

A slight modification of Fong's proof yields that for $d \geqslant 2, h(\chi)$ never exceeds $(d-2)$. Brauer and Feit [2] obtain this bound for an arbitrary finite group. In this paper we prove the following result.

TheOREM. Let $G$ be a finite p-soluble group with a block $B$ of defect $d \geqslant 2$. Then there exists a function $f(p, d)$ such that $h(\chi) \leqslant f(p, d)$ for all ordinary characters $\chi \in B$;

$$
f(p, d)= \begin{cases}\left(\frac{3 d-4}{4}\right) & \text { if } p=2, \\ \frac{\left(p^{2}+1\right)}{\left(p^{2}-p+1\right)}\left(\frac{d-1}{2}\right) & \text { if } p \text { is an odd Fermat prime, } \\ \frac{(p+1)}{(p)} \frac{(d-1)}{2} & \text { if } p \text { is any other prime. }\end{cases}
$$

We give examples to show that this bound is of the right order of magnitude.

Received by the editors March 28, 1975.

AMS (MOS) subject classifications (1970). Primary 20C20.

Key words and phrases. Modular representations, block, defect, height.

() American Mathematical Society 1977 
LEMMA 1. Let $G$ be a finite p-soluble group and $B$ be a block of $G$ with defect group $D$, of order $p^{d}$. Suppose that $H \triangleleft G$, then there exists an irreducible constituent of $\left.\chi\right|_{H}$, say $\theta$, such $h(\chi) \leqslant h(\theta)+\nu_{p}(|G: H|) . h(\theta)$ denotes the height of $\theta$ over the block $b$ of $H$ with $\theta \in b$ and $h(\chi)$ denotes the height of $\chi$ over $B$.

Proof. Choose a series $G=G_{1} \geqslant G_{2} \geqslant \cdots \geqslant G_{r}=H$ such that $G_{i+1}$ is a maximal normal subgroup of $G_{i}$ for $i=1, \ldots, r-1$. Define $e_{i}=\nu_{p}\left(\left|G_{i}\right|\right)$. Now choose ordinary characters $\chi_{1}, \ldots, \chi_{r}$ such that $\chi_{i}$ is an ordinary character of $G_{i}$ and $\chi=\chi_{1}$, and $\chi_{i+1}$ is an irreducible constituent of $\chi_{i}$ restricted to $G_{i+1}$. Now there exist blocks $B_{1}, \ldots, B_{r}$ with $B_{i}$ a block of $G_{i}$ containing $\chi_{i}$ for $i=1, \ldots, r$. Let $\theta=\chi_{r}$ and hence $B_{r}=b$. These conditions mean that $B_{i}$ covers $B_{i+1}$ in the sense of Brauer [1]. Finally let $D_{i}$ be a defect group of $B_{i}$ for each $i$ and suppose that $d_{i}=$ defect of $B_{i}$. We have

$$
\nu_{p}\left(\operatorname{deg} \chi_{i}\right)=e_{i}-d_{i}+h\left(\chi_{i}\right) \quad \text { for } i=1, \ldots, r \text {. }
$$

When $\left|G_{i}: G_{i+1}\right|$ is coprime to $p$ then by Cliffords theorem $\nu_{p}\left(\operatorname{deg} \chi_{i}\right)$ equals $\nu_{p}\left(\operatorname{deg} \chi_{i+1}\right)$. Clearly $e_{i}=e_{i+1}$ and by $[1,2 \mathrm{E}] d_{i}=d_{i+1}$. Thus $h\left(\chi_{i}\right)$ $=h\left(\chi_{i+1}\right)$. Otherwise $\left|G_{i}: G_{i+1}\right|=p$ and Cliffords theorem yields that

$$
\nu_{p}\left(\operatorname{deg} \chi_{i}\right) \leqslant \nu_{p}\left(\operatorname{deg} \chi_{i+1}\right)+1 \text {. }
$$

Also $e_{i}=e_{i+1}+1$ and by [3], $d_{i} \leqslant d_{i+1}+1$. We conclude that in this case $h\left(\chi_{i}\right)<h\left(\chi_{i+1}\right)+1$.

Hence $h(\chi)=h\left(\chi_{1}\right) \leqslant h\left(\chi_{r}\right)+\nu_{p}(|G: H|)=h(\theta)+\nu_{p}(|G: H|)$ as required.

LEMMA 2. If $G$ is a finite $p$-soluble group which is faithfully and irreducibly represented on a vector space $V$ of dimension $n$ over $G F(p)$ then $\nu_{p}(|G|)$ does not exceed $\lambda(p, n)$ where

$$
\lambda(p, n)= \begin{cases}(n-1) & \text { if } p=2 \\ (n p) /(p-1)^{2} & \text { if } p \text { is an odd Fermat prime } \\ n /(p-1) & \text { otherwise }\end{cases}
$$

Proof. $\nu_{2}(|G|) \leqslant n-1$ by Huppert [7, Satz 14]. For odd $p$ a paper of Winter [8] yields that

$$
\nu_{p}(|G|) \leqslant \begin{cases}\sum_{i=0}^{\infty} \frac{n}{p^{i}(p-1)} & \text { for } p \text { Fermat } \\ \sum_{i=1}^{\infty} \frac{n}{p^{i}} & \text { for } p \text { not Fermat. }\end{cases}
$$

Since for $|x|<1, \sum_{i=0}^{\infty} x^{i}=1 /(1-x)$ our lemma follows easily.

LEMMA 3. If $G$ is a finite $p$-soluble group with $O_{p^{\prime} p}(G) \cong O_{p^{\prime}}(G) \times O_{p}(G)$ and if $\left|O_{p}(G): \Phi\left(O_{p}(G)\right)\right|=p^{n}$ where $\left|O_{p}(G)\right|=p^{m}$ then

$$
\nu_{p}(|G|) \leqslant m+\lambda(p, n)
$$


Proof. By $[5,1.2 .5] G / O_{p^{\prime} p}(G)$ is faithfully represented on $O_{p^{\prime} p}(G) / F$ where $F / O_{p^{\prime}}(G)=\Phi\left(O_{p^{\prime} p}(G) / O_{p^{\prime}}(G)\right)$. Thus under our hypotheses $G / O_{p^{\prime} p}(G)$ is faithfully represented on $O_{p}(G) / \Phi\left(O_{p}(G)\right)$. Let $L_{1}, \ldots, L_{s}$ denote the $p$-chief factors of $G$ lying between $O_{p}(G)$ and $\Phi\left(O_{p}(G)\right)$. Then $C=$ $\cap{ }_{i=1}^{s} C_{G}\left(L_{i}\right) \geqslant O_{p^{\prime} p}(G)$ since $O_{p^{\prime} p}(G)$ centralizes all $p$-chief factors. For each $i=1, \ldots, s$ we have that $G / C_{G}\left(L_{i}\right)$ is a faithful irreducible subgroup of $G L\left(n_{i}, p\right)$ where $n_{i}$ is just the rank of $L_{i}$. Since $C / O_{p^{\prime} p}(G)$ is merely a group of automorphisms of a $p$-group which stabilizes a normal series for that group we conclude that $C / O_{p^{\prime} p}(G)$ is a $p$-group and thus $C=O_{p^{\prime} p}(G)$.

Now $G / O_{p^{\prime} p}(G)$ is isomorphic to a subgroup of $G / C_{G}\left(L_{1}\right) \times \cdots \times$ $G / C_{G}\left(L_{s}\right)$ so in particular

$$
\nu_{p}\left(\left|G: O_{p^{\prime} p}(G)\right|\right) \leqslant \sum_{1}^{s} \nu_{p}\left(\left|G: C_{G}\left(L_{i}\right)\right|\right) .
$$

Since $\lambda(p, k)$ is linear in the second variable, and using Lemma 2 we see that $\nu_{p}(|G|) \leqslant m+\lambda(p, n)$ as required.

Proof of TheOREM. We proceed by induction on the order of $G$. By [4, 2B and 2D] we may assume that all blocks of $G$ have maximal defect, so $d=\nu_{p}(|G|)$. Furthermore $O_{p^{\prime}}(G)$ is cyclic and central in $G$ so $O_{p^{\prime} p}(G)=$ $O_{p^{\prime}}(G) \times O_{p}(G)$. Let $H=O_{p}(G)$ and set $|H|=p^{m}$ and $|H: \Phi(H)|=p^{n}$. Let $\theta$ be an irreducible constituent of $\left.\chi\right|_{H}$. Now $\nu_{p}(|G: H|)=d-m$ and thus by Lemma $1 h(\chi) \leqslant h(\theta)+d-m$. We consider two possibilities:

(a) $H$ is abelian. In this case $h(\theta)=0$ and by Lemma 3 , since $n \leqslant m$ we have that $d \leqslant m+\lambda(p, m)$. When $p=2, \lambda(2, m)=m-1$ and so $d \leqslant 2 m-$ 1 . Hence $h(\chi) \leqslant(d-1) / 2$. For $p$ an odd Fermat prime a similar calculation yields $h(\chi) \leqslant(p d) /\left(p^{2}-p+1\right)$. Finally for $p$ odd and not Fermat, using Lemma 2 again we deduce that $h(\chi) \leqslant d / p$. These three bounds are less than the ones appearing in the statement of the theorem.

(b) $H$ is nonabelian. Now $h(\theta) \leqslant(m-1) / 2$ since $\theta$ is a character of a nonabelian $p$-group of order $p^{m}$. Also $H$ nonabelian implies that $n \leqslant m-1$. Thus $h(\chi) \leqslant d-(m+1) / 2$ by Lemma 1 and $d \leqslant m+\lambda(p, m-1)$ by Lemma 3. For $p=2, d \leqslant 2(m-1)$ and so $h(\chi) \leqslant(3 d-4) / 4$. When $p$ is an odd Fermat prime then $d \leqslant\left(m\left(p^{2}-p+1\right)-p\right) /(p-1)^{2}$ and thus $h(\chi)$ $\left.\leqslant\left(\left(p^{2}+1\right) /\left(p^{2}-p+1\right)\right)(d-1) / 2\right)$. Finally for non-Fermat primes $p$, $d \leqslant(m p-1) /(p-1)$ and a brief calculation yields

$$
h(\chi) \leqslant((p+1) / p)((d-1) / 2)
$$

Our theorem is now proved.

The theorem is best possible in the following sense: Given an odd integer $d \geqslant 1$ choose $p$ to be a prime with $p>d$. Now there exists an extraspecial $p$ group of order $p^{d}$; this group possesses an ordinary character of height $\frac{1}{2}(d-1)$. We have so chosen things that $\frac{1}{2}(d-1)$ is the greatest integer less than $f(p, d)$. We give less trivial examples for $p=2$ and $p=3$ below.

(1) Let $G_{i} \cong \operatorname{GL}(2,3)$, the group of $2 \times 2$ matrices over the Galois field of three elements, for $i=1,2, \ldots, n$. Form the central product $G(n)$ $=G_{1} Y G_{2} Y \cdots Y G_{n}$ (see [6, I.9.10]). Let $Z\left(G_{i}\right)=g p\left\{z_{i}: z_{i}^{2}=1\right\}$ then $G(n)$ $\cong\left(G_{1} \times G_{2} \times \cdots \times G_{n}\right) / \Delta$ where $\Delta=g p\left\{z_{1} z_{2}^{-1}, z_{2} z_{3}^{-1}, \ldots, z_{n} z_{1}^{-1}\right\}$. Now $G$ 
is a 2-soluble group and $O_{2^{\prime}}(G(n))=1$. Thus $G(n)$ possesses one block for the prime 2 and hence this has defect $d=3 n+1$. $G_{i}$ has a character $\theta_{i}$ of degree four such that $\theta_{i}\left(z_{i}\right)=-4$. Form $\theta_{1} \otimes \theta_{2} \otimes \cdots \otimes \theta_{n}=\chi$, an irreducible character of $G(n)$, since one easily checks that $\Delta \leqslant \operatorname{Ker} \chi$. We have that $h(\chi)=2 n=2(d-1) / 3$.

(2) Let $E$ be the extraspecial 3-group of order 27 and exponent 3. Since $\operatorname{SL}(2,3)$ is isomorphic to a subgroup of the automorphism group of $E$, we may form the semidirect product of $E$ by $\operatorname{SL}(2,3)$. Denote this group by $H$. $H$ has a centre of order 3 and furthermore has an irreducible character of degree 9, say $\alpha$. If $Z(H)=g p\left\{z_{i}: z_{i}^{3}=1\right\}$ then $\alpha\left(z_{i}\right)=9 \omega$ and $\alpha\left(z_{i}^{2}\right)=9 \omega^{2}$ where $\omega$ is a primitive cube root of unity. As in the previous example we construct $H(n)$, the central product of $n$ copies of $H . H(n)$ is a 3-soluble group in which $O_{3^{\prime}}(H(n))=1$ and so $H(n)$ has a unique block for the prime 3 and this has defect $d=3 n+1$. The character $\psi=$ tensor product of $n$ copies of $\alpha$, is an irreducible character of $H(n) . \psi$ has height $2 n=2(d-1) / 3$.

We have shown that if $g(p, d)$ is the precise bound on the heights of characters of $p$-soluble groups then

$$
\begin{aligned}
& 2(d-1) / 3 \leqslant g(2, d) \leqslant 3(d-1) / 4, \\
& 2(d-1) / 3 \leqslant g(3, d) \leqslant 5(d-1) / 7 .
\end{aligned}
$$

In fact there exist examples for all primes $p$ of $p$-soluble groups $G$ with a $p$-block $B$, of defect $d$, containing an ordinary character of height exceeding $(d-1) / 2$.

\section{REFERENCES}

1. R. Brauer, Some applications of the theory of blocks of characters of finite groups. IV, J. Algebra 17 (1971), 489-521. MR 43 \#7520.

2. R. Brauer and W. Feit, On the number of irreducible characters of finite groups in a given block, Proc. Nat. Acad. Sci. U.S.A. 45 (1959), 361-365.

3. P. Fong, Some properties of characters of finite solvable groups, Bull. Amer. Math. Soc. 66 (1960), 116-117. MR 22 \#2655.

4. - On the characters of p-solvable groups, Trans. Amer. Math. Soc. 98 (1961), 263-284. MR 22 \#11052.

5. P. Hall and G. Higman, On the p-length of p-soluble groups and reduction theorems for Burnside's problem, Proc. London Math. Soc. (3) 6 (1956), 1-42. MR 17, 344.

6. B. Huppert, Endliche Gruppen. I, Die Grundlehren der math. Wissenschaften, Band 134, Springer-Verlag, Berlin and New York, 1967. MR 37 \# 302.

7. - Linear auflösbare Gruppen, Math. Z. 67 (1957), 479-518. MR 19, 729.

8. D. L. Winter, p-solvable linear groups of finite order, Trans. Amer. Math. Soc. 157 (1971), 155-160. MR 43 \#2092.

Department of Pure Mathematics, University of Liverpool, Liverpool, Great Brtain

Current address: Department of Mathematics, Oxford Polytechnic, Oxford, Great Britain 